\title{
The analgesic efficacy of local infiltration analgesia versus peripheral nerve block after anterior cruciate ligament reconstruction: a systematic review and meta-analysis.
}

\author{
Eric Albrecht, ${ }^{1}$ Robin Martin, ${ }^{2}$ Alain Jacot-Guillarmod, ${ }^{1}$ Kyle Robert Kirkham ${ }^{3}$
}

1. Department of Anaesthesia, Lausanne University Hospital, Lausanne, Switzerland.

2. Department of Orthopaedic surgery, Lausanne University Hospital, Lausanne, Switzerland

3. Department of Anaesthesia, Toronto Western Hospital, University of Toronto, Toronto, Canada.

\section{Background and Goal of Study}

Many authors consider blockade of the femoral nerve distribution the gold standard analgesic treatment after anterior cruciate ligament reconstruction. However, some argue an alternative approach of local infiltration analgesia, using local anesthetics injection into the surgical site, has similar efficacy. The objectives of this meta-analysis were to compare the analgesia and functional outcomes of both treatments following anterior ligament reconstruction.

\footnotetext{
Materials and Methods

The methodology followed the PRISMA statement guidelines. The primary outcomes were pain scores at rest (analogue scale, $0-10)$ in the early ( $0-2$ postoperative hours), intermediate (3-12 postoperative hours), and late postoperative periods (13-24 postoperative hours) Secondary outcomes included range of motion, quadriceps muscle strength, and complication rates (neurologic events, cardiovascular events, falls, knee infections, or knee chondrolysis).
}

\section{Results and Discussion}

Ten trials, including 588 patients, were identified. Pain scores in the early (mean difference: $1.6 ; 95 \% \mathrm{CI}: 0.2,2.9 \mathrm{mg} ; \mathrm{I} 2=97 \% ; \mathrm{p}=0.02$ ), intermediate (mean difference: $1.2 ; 95 \% \mathrm{CI}: 0.4,1.5 ; \mathrm{I} 2=85 \% ; \mathrm{p}=0.002$ ) and late (mean difference: $0.7 ; 95 \% \mathrm{CI}: 0.1$, $1.4 ; \mathrm{I} 2=82 \% ; \mathrm{p}=0.03$ ) postoperative periods were significantly lower in patients who received a femoral nerve block (table 1). The quality of evidence for our primary outcomes were moderate to high according to the Grades of Recommendation, Assessment, Development, and Evaluation (GRADE) Working Group System. Regarding the functional outcomes, only one trial reported range of motion at 48 postoperative hours, which was found to be similar between groups (mean difference: $\left.-5^{\circ} ; 95 \% \mathrm{CI}:-14,4^{\circ} ; \mathrm{p}=0.27\right)$. No trials sought to record side-effects such as neurologic events, cardiovascular events, falls, knee infections, or knee chondrolysis.

\section{Conclusion}

Femoral nerve block provides superior postoperative analgesia after anterior cruciate ligament reconstruction when compared to local infiltration analgesia. The impact of improved analgesia on function remains unanswered due to the lack of functional outcomes reported in existing literature.

Table 1. Pain scores at rest in the early, intermediate and late postoperative periods (analogue scale, 0 - 10). CI, confidence interval; $n$, number of events; $\mathbf{N}$, total number of participants; SD, standard deviation.

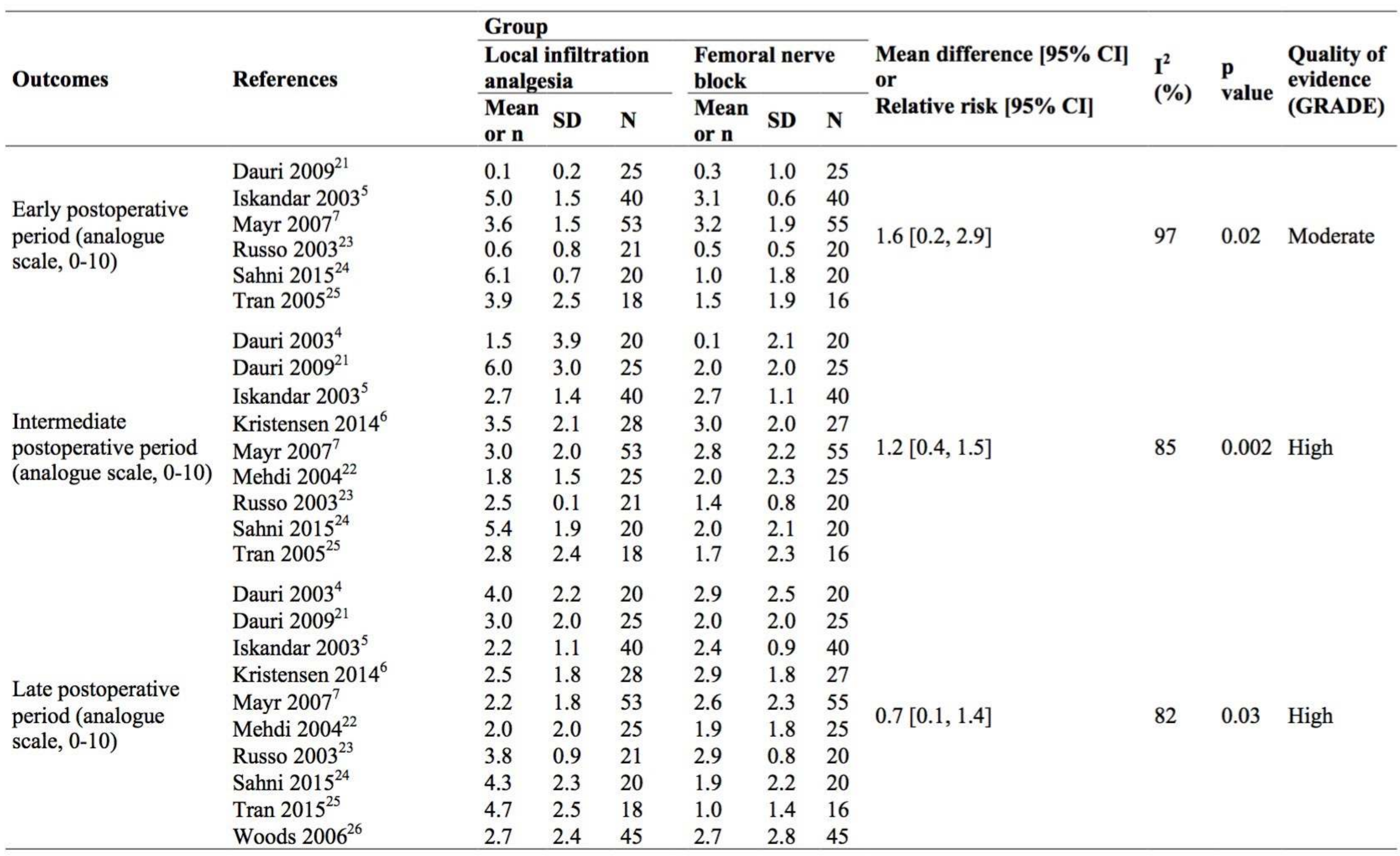

